\title{
The Utility of Picosecond Nd:YAG Laser for Tattoo Removal
}

\author{
Kyong Chan Park \\ Eun Soo Park \\ Seung Min Nam \\ Jin Su Shin
}

Department of Plastic and Reconstructive Surgery, Soonchunhyang University Bucheon Hospital, Bucheon, Korea
Received December 11, 2020

Accepted February 16, 2021

\footnotetext{
Correspondence

Eun Soo Park

Department of Plastic and Reconstructive Surgery, Soonchunhyang University Bucheon Hospital, 170 Jomaru-ro, Bucheon 14584, Korea Tel.: +82-32-621-5319

Fax: +82-32-621-5016

E-mail: peunsoodschmc.ac.kr

(c) Korean Society for Laser Medicine and Surgery

(c) This is an open access article distributed under the terms of the Creative Commons Attribution NonCommercial License (http://creativecommons.org/ licenses/by-nc/4.0) which permits unrestricted noncommercial use, distribution, and reproduction in any medium, provided the original work is properly cited.
}

\author{
Background and Objectives \\ Several predicted optimal parameters for laser treatment have been \\ suggested, making the settings subject to variation. Thus, picosecond \\ lasers may require more data and studies to optimize the laser \\ parameters, increase the efficacy of each session, and minimize the total \\ number of sessions. This study evaluated the results of picosecond laser \\ tattoo removal in variable locations, focusing on the aesthetic outcome, \\ number of procedures, and adverse effects.
}

\section{Materials and Methods}

Nine patients who underwent picosecond laser treatment from February 2014 to July 2020 were enrolled. Before the procedures, the required number of sessions was estimated using the Kirby-Desai scale. At the end of the treatment, the patients assessed their satisfaction. The patient and two plastic surgeons assessed the clearance of the tattoo.

\section{Results}

The mean of the clearance was $86.6 \%$ in both the patient and investigators assessment. The patients reported satisfaction for an average score of $5.1 \pm 0.78$ for the aesthetic outcome, $4.5 \pm 0.78$ for pain, $4.1 \pm 0.92$ for the number of procedures, and $4.7 \pm 0.97$ for adverse effects. The average number of actual procedures was $6.7 \pm 1.20$. The average Kirby-Desai score was $7.1 \pm 1.45$. Their correlation coefficient was 0.803 , which is considered a strong positive correlation.

\section{Conclusion}

Picosecond lasers have a remarkable ability to degrade smaller tattoo pigments through a photoacoustic effect. Moreover, a picosecond laser treatment for tattoo removal can be a safe and effective method. Picosecond lasers are a promising technology with the potential to optimize the treatment of tattoos.

\section{Key words}

Laser therapy; Tattoo removal; Kirby-Desai scale; Picosecond laser 


\section{INTRODUCTION}

Since ancient times, tattoos have been used to express an artistic perspective, enhance beauty, demonstrate uniqueness and signify belonging or punish. A rise in US adult tattoo prevalence was reported from $16 \%$ in 2003 to $21 \%$ in 2012.' Like western countries, tattooing has been gaining popularity in Korea. ${ }^{2}$ With the increasing popularity of tattooing, the increasing numbers of people are also requiring tattoo removal. Lasers were first used to remove tattoos in the 1960s. ${ }^{3}$ Selective photothermolysis, first described in the early 1980s, which is the concept that when a chromophore is heated for shorter than its thermal relaxation time, selective destruction of that chromophore can occur without damaging surrounding tissue. ${ }^{4}$ To achieve selective photothermolysis, sufficient energy is needed to be delivered with a pulse duration which is shorter than the thermal relaxation time, defined by the size and shape of the target. The smaller the target is, the shorter the thermal relaxation time would be and therefore the shorter laser pulse duration is required. In the case of tattoo, the ink is the chromophore. The pigment is very small, so the thermal relaxation time is less than 10 nanoseconds, suggesting that rapid heating with short pulse duration, in the picosecond range, should be effective..$^{5-7}$ A wide range of predicted optimal parameters for laser treatment have been suggested, and it make the settings and parameters subject to be variable. Thus, picoseconds lasers may require more data and studies to optimize laser parameter, increase the efficacy of each sessions, and minimize the total number of sessions.

The purpose of this study was to evaluate the results of picosecond tattoo removal in variable location, focused on aesthetic outcome, the number of procedures and adverse effects.

\section{MATERIALS AND METHODS}

\section{Patients and data extraction}

We performed a retrospective analysis on 9 patients who underwent tattoo removal using picosecond laser between February 2014 and July 2020. A senior surgeon (E.S.P.) performed the procedures at Soonchunhyang University Bucheon Hospital. We excluded the patients with a history of keloidal scarring, pregnancy, infection of wound or the administration of photosensitive drugs. Patients who did not have at least 6 months of postoperative follow-up were excluded. All outcome data including complication and aesthetic outcome were reviewed before and after the picosecond laser procedure.

This study was approved by the institutional review board and conformed to the Declaration of Helsinki. Informed consents were obtained from all patient for both the laser procedure and the publication of the results including photographs.

\section{Pre-treatment preparation}

Before the laser procedure, all patients applied a 5\% lidocaine topical anesthetic ointment (Emla ${ }^{\circledR}$; AstraZeneca AB, Karlskoga, Sweden) to the target area for 30 minutes. This topical ointment was washed off with soap and water before the procedure. Protective eyewear was properly placed on the patients.

\section{Laser procedure}

All of laser procedure was performed with picosecond laser (PICOCARE ${ }^{\circledR}$; WONTECH, Daejeon, Korea). Treatment was performed with a wavelength of $532 \mathrm{~nm}$ for red colored tattoo in one case or 1,064 nm picosecond for dark colored tattoo for the rest. Single procedure with one pass were performed using a spot size of from $4 \mathrm{~mm}$, fluence of 2.5 to $4.8 \mathrm{~J} / \mathrm{cm}^{2}$, repetition rate of 6 to $10 \mathrm{~Hz}$. The parameters were adjusted based on the size and type of tattoo and patient's skin type. The lower fluence was

Table 1. Kirby-Desai scale

\begin{tabular}{|c|c|c|c|c|c|c|}
\hline Point & Phototype & Location & Color & Ink amount & Scarring & Layering \\
\hline 0 & & & & & None & None \\
\hline 1 & I & Head, neck & Black & Amateur & Minimal & \\
\hline 2 & II & Upper trunk & Mostly black with some red & Minimal & & Layering \\
\hline 3 & III & Lower trunk & Mostly black and red with some other colors & Moderate & Moderate & \\
\hline 4 & IV & Proximal extremity & Multiple colors & Significant & & \\
\hline 5 & V & Distal extremity & & & Significant & \\
\hline 6 & VI & & & & & \\
\hline
\end{tabular}

Six numerical parameters: skin type, location, color, amount of ink, scarring and layering.

Parameter scores are added to yield a combined score showing the estimated number of treatments needed for successful tattoo removal. 
usually progressively increased on each subsequent procedure. The number of procedures was dependent on the rate of clearance and the physician's discretion. Intervals between sessions were determined individually about $6 \pm$ 2 weeks, depending on the stage and results.

\section{Post-treatment care}

Cold compresses were used to minimize discomfort and an antibiotic ointment or ointment-based emollients was applied to the target area after each laser treatment session. The patients were educated to avoid direct sunlight and recommended to apply a sunscreen agent between the laser treatment sessions. We educated the patients to visit hospital immediately when they had any adverse effect.

\section{Assessment}

Prior to initiation of the treatment, a Kirby-Desai score was assessed for each tattoo based on patient's history and photographs. In the Kirby-Desai scale, parameter scores were added to yield a total score needed for prediction of the required number of sessions, consisting of six parameters: skin type, location, color, amount of ink, scarring and layering (Table 1). Tattoos scoring greater than 15 points may be difficult to remove.

At the end of the treatment, the patients were asked to rate their satisfaction for the aesthetic outcome $11=$ dissatisfactory, $6=$ satisfactory), pain $(1=$ worst pain, $6=$ no pain), adverse effect ( 1 = dissatisfactory, 6 = satisfactory) and the number of procedure $11=$ dissatisfactory, $6=$ satisfactoryl using a 6-point scale. The category of adverse effect included long-term effects such as hyper- or hypopigmentation or color change.

Standardized photographs were taken before the procedure and after every subsequent procedure. The photographs were assessed by 2 plastic surgeons, who were blinded for patient information, for the percentage of clearance, where $0 \%$ was an absence of clearance and $100 \%$ was a complete removal of tattoo. And the photographs were grossly evaluated by the patient himself with regarding to clearance.

\section{Statistical analysis}

All analyses were performed with IBM Statistical Package for the Social Sciences (version 24.0; IBM Corp., Armonk, NY, USA). We calculated the Pearson correlation coefficient between Kirby-Desai scores and the actual number of sessions, which showing the correlation between the two groups. We consider the correlation coefficient values $>0.8$ as strongly positive correlation.

\section{RESULTS}

A total of 9 patients underwent picosecond laser tattoo removal, and their ages ranged from 24 to 52 years (mean,

Table 2. Patient characteristics and number of procedures

\begin{tabular}{lllcc}
\hline \multirow{2}{*}{ Sex/Age, $y$} & \multicolumn{3}{c}{ Tattoo } & \multicolumn{2}{c}{$\begin{array}{c}\text { Procedure, } \\
\text { no. }\end{array}$} & Predicted \\
no. of Tx.
\end{tabular}

AC, almost complete.

*The calculated Kirby-Desai scores.
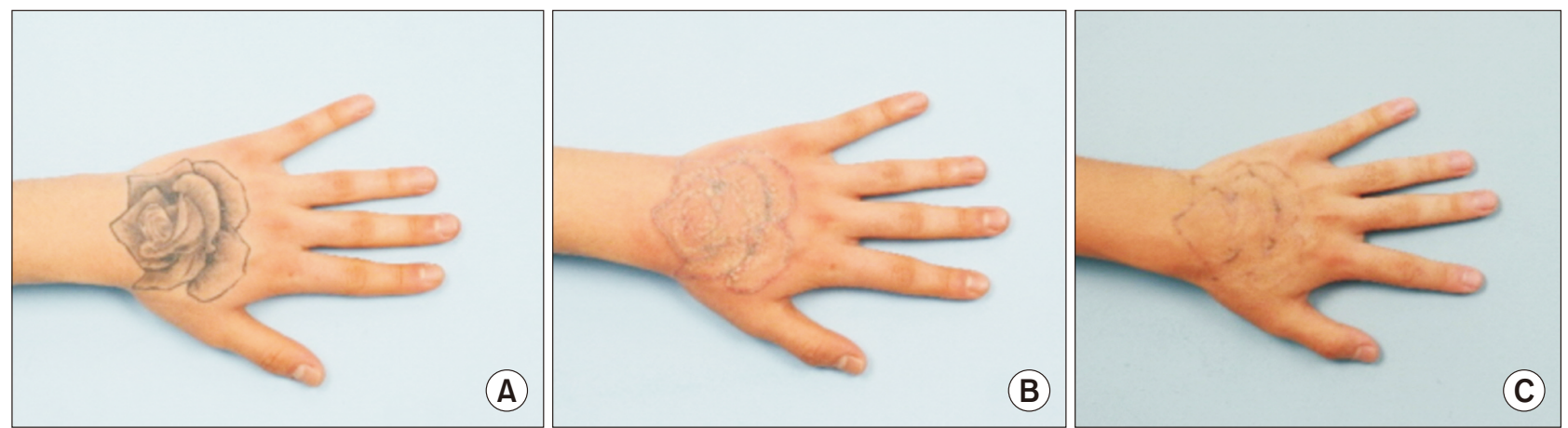

Fig. 1. Clinical photographs A 31-year-old female patient with tattoo on left hand consisting of single black color. (A) Prior to the procedure. (B) Immediately after the first procedure of tattoo removal, whitening results prevents subsequent pulses from reaching the remaining underlying pigments are shown. (C) After the final 4th session, for 1 year, there was $80 \%$ clearance of peripheral portion, and almost complete clearance of central portion. 


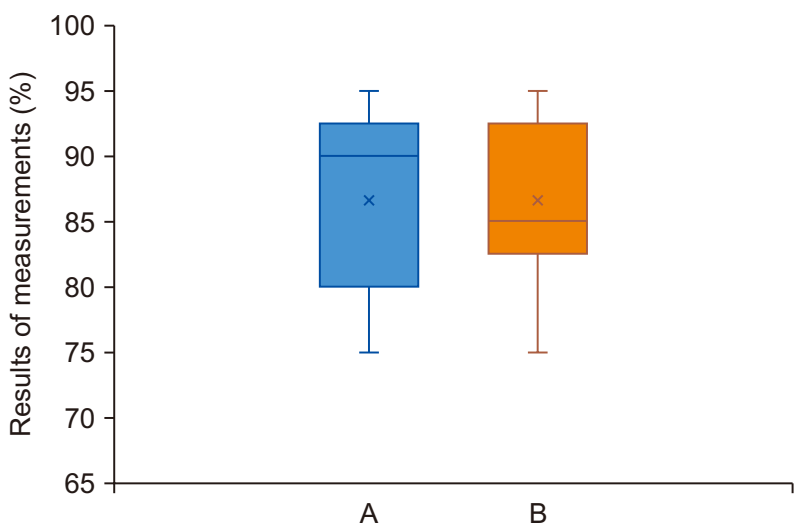

Fig. 2. Tattoo removal clearance Assessment of tattoo removal clearance by patient (A, blue) and investigator (B, orange) The mean of the clearance was $86.6 \%$ (IQR $80-92.5$ ) in patient group, and $86.6 \%$ (IQR $82.5-92.5$ ) in investigator group. IQR, interquartile range.

35 years). 5 women (56\%) and 4 men (46\%) were treated. One of 9 patients were single red colored tattoos and black tattoos were shown in rest of cases (Table 2). All of 9 patients (100\%) had clearance of greater than $75 \%$, and 7 (78\%) obtained almost complete clearance (Fig. 1). The mean of the clearance was $86.6 \%$ linterquartile range [IQR] 80-92.5) in patient and $86.6 \%$ (IQR 82.5-92.5) in investigator. (Fig. 2). The patients reported satisfaction average score for the aesthetic outcome of 5.1 with a standard deviation (SD) of \pm 0.78 . The average score \pm SD was $4.5 \pm$ 0.78 for pain, $4.1 \pm 0.92$ for the number of procedures and $4.7 \pm 0.97$ for adverse effect.

The average number of actual procedures was $6.7 \pm$ 1.20. This result correlates with the average Kirby-Desai score of $7.1 \pm 1.45$. The correlation coefficient was 0.803 between the Kirby-Desai score and the actual number, which we consider as strongly positive correlation (Table 3).

\section{DISCUSSION}

Picosecond lasers, with pulse duration of $10^{-12}$ seconds, are emerged as fast and effective method for tattoo removal because most tattoo pigment size ranges from 30 to $300 \mathrm{~nm}$ with short thermal relaxation time $(<10 \mathrm{~ns}){ }^{8}$ Therefore, picosecond lase can provide greater thermal stress in targeted tattoos. ${ }^{9}$ In addition to photoacoustic effects within targeted tattoos leading to mechanical dissolution of the ink particles, endothermic steam carbon reaction occurs, which change the properties of inks, reducing their visibility. ${ }^{10,11}$ These mechanisms of picoseconds laser tattoo removal have verified by computer
Table 3. Assessment by patient

Post-treatment (median)

$\begin{array}{lc}\text { Patient's satisfaction } & \\ \text { Aesthetic outcome } & 5.1 \pm 0.78 \\ \text { Pain } & 4.5 \pm 0.78 \\ \text { No. of procedures } & 4.1 \pm 0.92 \\ \text { Adverse effects } & 4.7 \pm 0.97 \\ \text { The number of sessions } & \\ \text { Kirby-Desai scores } & 7.1 \pm 1.45 \\ \text { Actual numbers } & 6.7 \pm 1.20 \\ \text { *Correlation coefficient } & 0.803\end{array}$

Data are presented as mean \pm standard daviation.

*Pearson correlation coefficient for the relation of the Kirby-Desai scores and the actual numbers.

simulation. ${ }^{12}$ Furthermore, picosecond technology could permit lower fluences to be delivered, which theoretically lower the risk of adverse effect.?

Ross et al. was first to report the better effect of the picosecond laser comparing nanosecond laser in human patient using a 1,064-nm Nd:YAG laser with a pulse duration of either 35 picoseconds or 10 nanoseconds. The parameters included a fluence of $0.65 \mathrm{~J} / \mathrm{cm}^{2}$ and spot size of $1.4 \mathrm{~mm} .{ }^{5}$ Izikson et al reported using Yorkshire pig model that the 758-nm 500-picosecond laser with 3 different parameter settings provided greater tattoo clearance than quality-switched alexandrite laser with single setting (fluence of $8 \mathrm{~J} / \mathrm{cm}^{2}$, spot size of $3 \mathrm{~mm}$, and a pulse duration of 30 to 50 nanoseconds) in black tattoos. ${ }^{7}$ Choi et al. compared picosecond and nanosecond lasers in their ability to remove multi-colored tattoo using Hartley guinea pig. They first compared a nanosecond qualityswitched Nd:YAG laser with picosecond alexandrite and quality-switched $\mathrm{Nd}: Y A G$ lasers and then the picosecond quality-switched $\mathrm{Nd}: Y A G$ laser with the picosecond alexandrite laser, and concluded that picosecond lasers are more effective and safer than nanosecond laser. ${ }^{13}$

A wide range of predicted optimal parameters have been suggested, which make the settings and parameters subject to variation. In our study, the patients received variable times of picosecond laser in variable parameter setting, adjusted for the tattoo characteristics and patient's skin type.

The Kirby-Desai scale was first introduced in 2009 and has been validated and extensively described in the literatures. ${ }^{14}$ This scale can be used before the treatment to determine the number of treatments required for tattoo removal, decreasing the uncertainty of the laserremoval process. We used Kirby-Desai scores for assess the number of sessions, and the correlation between the 
estimated number and the actual number was strongly positive (0.803). Patients rated the number of procedures score of 4.1, which is relatively low score comparing with other subjective results, although of constant effort to optimize laser parameter and minimize the total number of sessions. However, this estimation was positive for them because it used to exceed the patient expectation, so did not over promise.

Adverse effects included pain, swelling and blistering, but resolved within few days, and it is related to the high score in patient satisfaction for adverse effect. We concerned hyperpigmentation or hypopigmentation, as reported as $20 \%$ of hypopigmentation and $13 \%$ of hyperpigmentation in previous study using picosecond alexandrite laser. ${ }^{15}$ However, we observed slight hypopigmentation in one case. In the study by Kirby et al., most of undesired pigment alterations were transient. ${ }^{16}$ The patients rated the pain score of 4.5, which is relatively satisfactory. All of patients were uniformly received topical anesthesia for 30 minutes, leading to sufficient anesthetic effect.

This study aimed to determine the efficacy of picosecond laser treatment in the tattoo removal. We evaluated the outcome with patients' subjective assessment and predicted the number of procedures using Kirby-Desai scale. The results of picosecond laser treatment were satisfactory for patients and assessed for successful by investigator.

Limitations of our study should be noted. First, we had small sample size. Even though we used distribution-free statistic method, further studies including larger sample size are needed. Second, there was no control group in this study. Prospective, controlled, and blinded studied are need for further evaluation. Third, the assessment for clearance of tattoo removal is based on photographic evaluation. Even though we educated how to evaluate the clearance by showing reference pictures, this method could be subjective.

\section{CONCLUSION}

Picosecond lasers demonstrate a greater ability to degrade smaller tattoo pigments through the photoacoustic effect. We provide evidence that the use of picosecond laser treatment for tattoo removal can be a safe and very effective method. Picosecond lasers are a promising technology with the potential to optimize the treatment of tattoos.

\section{CONFLICT OF INTEREST}

Eun Soo Park is an editorial board member of the journal but was not involved in the review process of this manuscript. Otherwise, there is no conflict of interest to declare.

\section{FUNDING}

This work was supported by the Soonchunhyang University Research Fund.

\section{REFERENCES}

1. Ho SG, Goh CL. Laser tattoo removal: a clinical update. J Cutan Aesthet Surg 2015;8:9-15.

2. Kim SY. A study on adult women's cosmetic tattoo experiences and comparison of health concern and health practice between the cosmetic tattooed and non-cosmetic tattooed groups. J Korean Acad Community Health Nurs 2017;28:69-77.

3. Goldman L, Rockwell RJ, Meyer R, Otten R, Wilson RG, Kitzmiller KW. Laser treatment of tattoos. A preliminary survey of three year's clinical experience. JAMA 1967;201:841-4.

4. Anderson RR, Parrish JA. Selective photothermolysis: precise microsurgery by selective absorption of pulsed radiation. Science 1983;220:524-7.

5. Ross V, Naseef G, Lin G, Kelly M, Michaud N, Flotte TJ, et al. Comparison of responses of tattoos to picosecond and nanosecond Q-switched neodymium: YAG lasers. Arch Dermatol 1998;134:167-71.

6. Herd RM, Alora MB, Smoller B, Arndt KA, Dover JS. A clinical and histologic prospective controlled comparative study of the picosecond titanium:sapphire $(795 \mathrm{~nm})$ laser versus the Q-switched alexandrite (752 nm) laser for removing tattoo pigment. J Am Acad Dermatol 1999;40:603-6.

7. Izikson L, Farinelli W, Sakamoto F, Tannous Z, Anderson RR. Safety and effectiveness of black tattoo clearance in a pig model after a single treatment with a novel $758 \mathrm{~nm} 500$ picosecond laser: a pilot study. Lasers Surg Med 2010;42:640-6.

8. Keaney TC, Alster TS. Tattoos and beyond: the clinical evolution of picosecond laser technology. Curr Dermatol Rep 2016;5:21721.

9. Luebberding S, Alexiades-Armenakas M. New tattoo approaches in dermatology. Dermatol Clin 2014;32:91-6.

10. Chen H, Diebold G. Chemical generation of acoustic waves: a giant photoacoustic effect. Science 1995;270:963-6.

11. Freedman JR, Kaufman J, Metelitsa Al, Green JB. Picosecond lasers: the next generation of short-pulsed lasers. Semin Cutan Med Surg 2014;33:164-8.

12. Ho DD, London R, Zimmerman GB, Young DA. Laser-tattoo 
removal--a study of the mechanism and the optimal treatment strategy via computer simulations. Lasers Surg Med 2002;30:389-97.

13. Choi MS, Seo HS, Kim JG, Choe SJ, Park BC, Kim MH, et al. Effects of picosecond laser on the multi-colored tattoo removal using Hartley guinea pig: a preliminary study. PLoS One 2018;13:e0203370.

14. Kirby W, Desai A, Desai T, Kartono F, Geeta P. The Kirby-Desai scale: a proposed scale to assess tattoo-removal treatments. J Clin Aesthet Dermatol 2009;2:32-7.

15. Saedi N, Metelitsa A, Petrell K, Arndt KA, Dover JS. Treatment of tattoos with a picosecond alexandrite laser: a prospective trial. Arch Dermatol 2012;148:1360-3.

16. Kirby D, Koriakos A, Desai A, Desai T. Undesired pigmentary alterations associated with $\mathrm{Q}$-switched laser tattoo removal: a retrospective study and review of the literature. Skin Aging 2010;18:38-40.

How to cite this article: Park KC, Park ES, Nam SM, Shin JS. The utility of picosecond Nd:YAG laser for tattoo removal. MedLasers2021;10:31-36.https://doi.org/10.25289/ML.2021.10.1.31 